Braz J Med Biol Res, October 2011, Volume 44(10) 1013-1017

doi: 10.1590/S0100-879X2011007500114

\section{New multilocus sequence typing of MRSA in São Paulo, Brazil}

M.S. Carmo, F. Inoue, S.S. Andrade, L. Paschoal, F.M. Silva, V.G.S. Oliveira and A.C.C. Pignatari

The Brazilian Journal of Medical and Biological Research is partially financed by

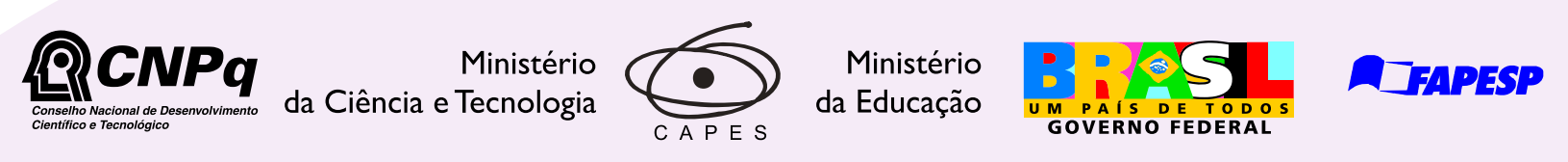

Institutional Sponsors
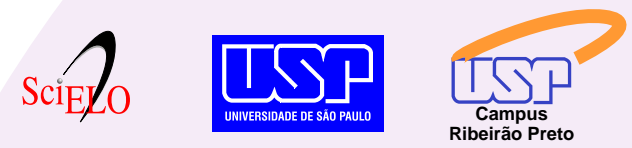

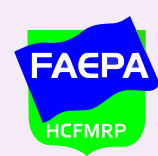

Ф SHIMADZU

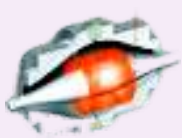

Explore High - Performance MS Orbitrap Technology In Proteomics \& Metabolomics

$\underset{\text { analitica }}{\text { analiticaweb.com.br }}$ SCIENTIFIC 


\title{
New multilocus sequence typing of MRSA in São Paulo, Brazil
}

\author{
M.S. Carmo ${ }^{1,2}$, F. Inoue ${ }^{1}$, S.S. Andrade ${ }^{1}$, L. Paschoal ${ }^{1}$, F.M. Silva ${ }^{1}$, \\ V.G.S. Oliveira ${ }^{1}$ and A.C.C. Pignatari ${ }^{1}$ \\ ${ }^{1}$ Laboratório Especial de Microbiologia Clínica, Departamento de Doenças Infecciosas e Parasitárias, \\ Universidade Federal de São Paulo, São Paulo, SP, Brasil \\ ${ }^{2}$ Centro Universitário Fundação Instituto de Ensino para Osasco, Osasco, SP, Brasil
}

\begin{abstract}
An increased incidence of nosocomial and community-acquired infections caused by methicillin-resistant Staphylococcus aureus (MRSA) has been observed worldwide. The molecular characterization of MRSA has played an important role in demonstrating the existence of internationally disseminated clones. The use of molecular biology methods in the surveillance programs has enabled the tracking of MRSA spread within and among hospitals. These data are useful to alert nosocomial infection control programs about the potential introduction of these epidemic clones in their areas. Four MRSA blood culture isolates from patients hospitalized at two hospitals in the city of São Paulo, Brazil, were analyzed; one of them was community acquired. The isolates were characterized as SCCmec, mecA and PVL by PCR, pulsed-field gel electrophoresis (PFGE) profile and molecular sequence typing (MLST) genotyping. The isolates presented type IV SCCmec, and none proved to be positive for $P V L$. The isolates showed a PFGE profile similar to the pediatric clone. MLST genotyping demonstrated that the isolates belonged to clonal complex 5 (CC5), showing a new yqiL allele gene, resulting in a new sequence typing (ST) (1176). Our results showed that strains of MRSA carrying a new ST are emerging in community and nosocomial infections, including bacteremia, in São Paulo, Brazil.
\end{abstract}

Key words: Methicillin-resistant Staphylococcus aureus; Molecular sequence typing; Resistance; Molecular typing

\section{Introduction}

Staphylococcus aureus has been the most important pathogen related to nosocomial infections in Brazil and remains the main agent isolated from community-acquired infections in most countries (1). Among species of Staphylococcus, they account for 30 to $50 \%$ of nosocomial infections and this number is on the rise $(2,3)$. After the description of the Brazilian epidemic clone (BEC) in 1992 (4), other studies further demonstrated the persistence of this microorganism in Brazilian hospitals (3). In these studies, the molecular characterization of methicillin-resistant $S$. aureus (MRSA) has been conducted primarily by pulsed-field gel electrophoresis (PFGE) and plasmid analysis, which showed the existence of internationally disseminated clones, reflecting epidemiological and geographical relatedness (5). The ability to correctly identify the MRSA strains is important for epidemiological understanding and decision making in public health (6).

Genetic structure models of bacterial populations were demonstrated in 1998 when the multilocus sequence typing (MLST) technique was introduced (http://www.mlst.net/). Molecular biology methods in monitoring programs have permitted the determination of inter- and intra-hospital spread of MRSA. Moreover, the MLST data obtained can be used to answer basic questions about the evolution and biology of the population of bacterial species (7). Such information can be of help, for example, in the development of more efficient vaccines against pathogenic microorganisms or for the planning and implementation of epidemiological measures. The generated data can be useful to alert the committees of nosocomial infection control about the introduction of potentially epidemic clones in their areas. The aim of the present study was to detect MRSA gene rearrangements with subsequent acquisition or loss of genetic determinants through different molecular typing techniques, including SCCmec typing, PFGE, and MLST.

Correspondence: A.C.C. Pignatari, Disciplina de Doenças Infecciosas, UNIFESP, Rua Leandro Duprat, 189, 04024-002 São Paulo, SP, Brasil. Fax: +55-11-5081-2819. E-mail: pignatari@terra.com.br

Received March 6, 2011. Accepted August 17, 2011. Available online September 2, 2011. Published October 10, 2011. 


\section{Material and Methods}

The study was carried out using selected bloodstream MRSA isolates obtained from a patient with communityacquired pneumonia admitted to a private general hospital in 2006 (A26151), from 2 patients with nosocomial infection admitted to a public university hospital in 2004 (A13357 and A14711), and from a patient with nosocomial infection admitted to a public general hospital in 2007 (A30988). The identification of $S$. aureus was confirmed by positive DNAse and tube coagulase tests. Resistance to methicillin was determined according to Clinical and Laboratory Standard Institute guidelines (8). All strains were tested for the presence of the nuclease (nuc) and mecA genes. DNA was isolated from the reference strain by the method of van Soolingen et al. (9). SCCmec was determined by the multiplex PCR protocol developed by Oliveira and de Lencastre (10). All samples previously classified as SCCmec type IV were subjected to PCR for the detection of the lukF-LUKS-PV and PV genes encoding the virulence factor PVL (Panton-Valentine leukocidin) (11). PFGE of total DNA was performed as described elsewhere (3). Global and local representative MRSA clones were included in the experiments. BEC, SCCmec type I (NCTC 10422), SCCmec type II (N315), SCCmec type III (85/2082), SCCmec type IVa (JCSC 1968), WB72 (USA300/ST8), WB49 (OSPC/ST30), and 481 (USA400/ST1) were used as control isolates.

PFGE patterns were compared using the BioNumerics version 5.0 software (Applied Maths, Belgium). The PFGE profiles of MRSA were classified as clone "A" with subtypes
"A1" and "A2", sharing more than $80 \%$ of Dice coefficient similarity between them. The dendrogram was constructed using the unweighted pair-group with arithmetic mean (UPGMA) method algorithm for phylogenetic analysis. The molecular typing by MLST was based on the sequence of seven housekeeping genes according to procedures previously described (12). The resulting sequences were analyzed and compared with those deposited in the database at the http://saureus.mlst.net/site. Using the START program and eBURSTv2 at http://pubmlst.org/software/ analysis/start, a dendrogram on sequence typing (ST) was built based on the data bank of each sample to illustrate the sample relationship and also to separate it into groups, representing clonal complexes, when available (13).

\section{Results and Discussion}

The MRSA samples studied here were resistant to oxacillin and showed the presence of the nuc and mecA genes. SCCmec determination showed that the A14711, A13357 and A26151 samples were SCCmec type IVa, and the A30988 sample was SCCmec type II.

All samples classified as SCCmec type IV were examined for the detection of the PVL virulence factor by PCR and were shown to be negative (data not shown).

The A13357 and 14711 isolates showed $85.7 \%$ similarity despite a difference in the yqiL allele and both had $82.9 \%$ similarity compared to the A26151 isolate. These isolates showed $76.7 \%$ similarity to the pediatric clone (Figure 1). The dendrogram constructed using the isolates character-

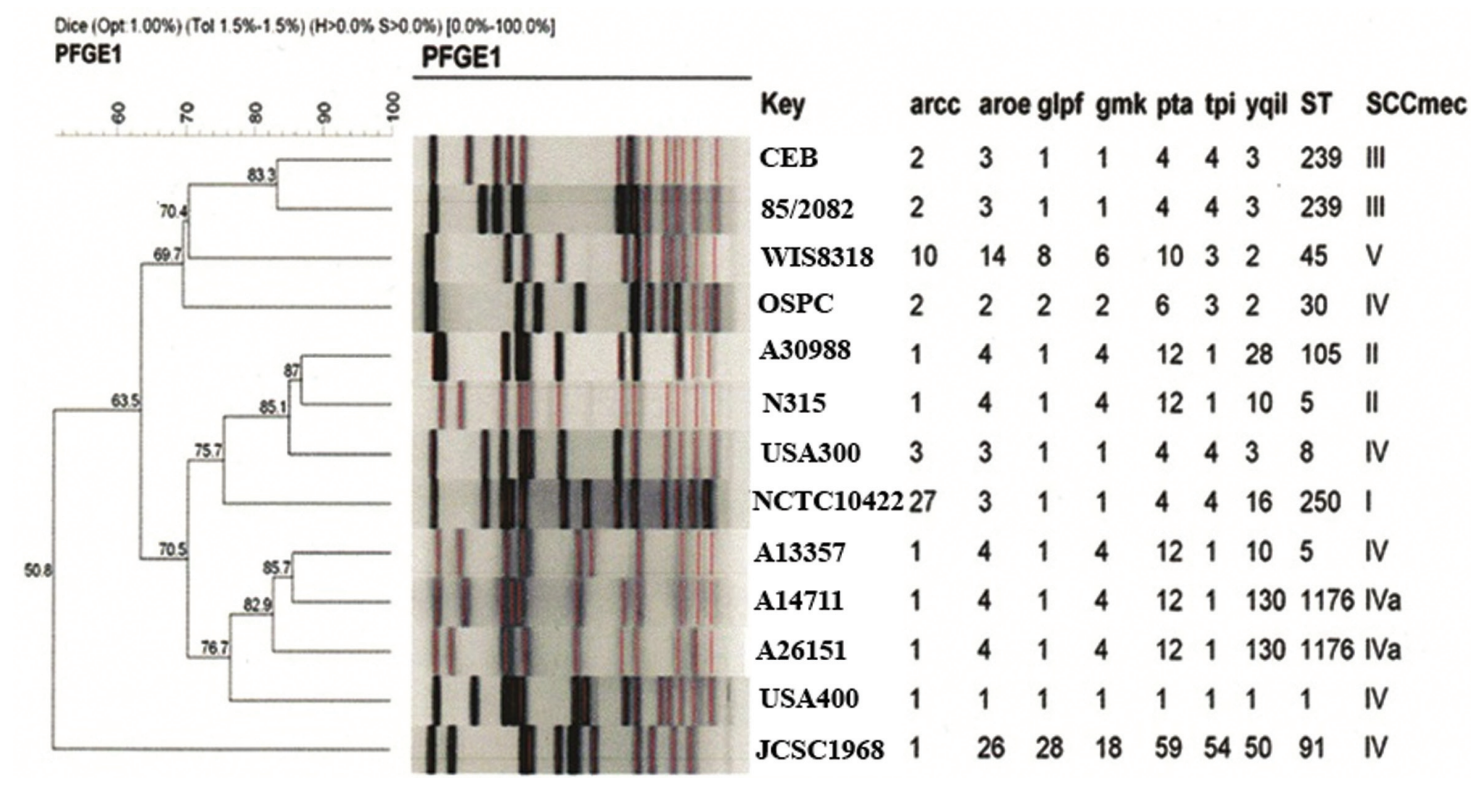

Figure 1. Pulsed-field gel electrophoresis (PFGE) dendrograms representing genetic relatedness using the Dice coefficient similarity and unweighted pair-group with arithmetic mean (UPGMA) method. Also included are the international methicillin-resistant Staphylococcus aureus (MRSA) clones. The right dendrogram columns indicate the number of alleles, sequence typing (ST) and SCCmec type, respectively. 
ized in the present study revealed more than $82 \%$ similarity between the $A 13357, A 26151$, and $A 14711$ samples. When these samples were compared to the $A 30988$ isolate the similarity was $70.5 \%$ and to the BEC $50.8 \%$.

MLST was identified by the PCR amplification of the $S$. aureus housekeeping genes in genomic DNA and resulted in approximately 500-bp fragments. For each isolate, the sequences obtained at each locus were compared to the database, and the alleles were numbered consecutively. Sequences were assigned as distinct alleles even though they differed at a single nucleotide site. Based on the numbers of the seven alleles identified for each sample, they received a number representing the ST. The evolutionary origins were examined by the eBURST algorithm in order to group the received allelic profiles into clonal complexes. The grouping was based on the similarities between sequence types at seven loci and was defined as a set sharing seven loci with any other in the data set.

The genetic similarity among isolates was also analyzed by the neighbor joining method. In this case, the allele nucleotide sequences of the seven genes were grouped into a single 3298-bp nucleotide sequence for each isolate. The sequences of different isolates were compared by determining the different nucleotide number between each sequence, for example in the genetic distance between isolates. These distances are represented in the gene tree branches constructed by this method (Figure 2). It was observed that the A30988 isolate was located in the same genogroup with a genetic distance of $86.4 \%$ showing similarity of $85.7 \%$ to A26151, A14711, A13357, and the pediatric clone. There is a clear bifurcation dividing the isolates belonging to the Brazilian epidemic clone, located at the bottom of the tree showing $86.1 \%$ similarity.

We found 4 different alleles for the glpF and tpi genes, 5 alleles for the arc $C$ and gmk genes, 6 alleles for the aro $E$ and pta genes, and 8 alleles for the yqil gene. The polymorphic sites between nucleotides ranged from 7 (tpi gene) to 12 (yqiL gene). Analysis of the eBURSTV2 allelic frequency of the $S$. aureus samples studied showed that the most common alleles were number 1 for the arc $C, g / p F, g m k$, and tpigenes $(50,78.6,35.7$, and $50 \%$, respectively), number 4 for the aroE gene (42.9\%), number 12 for the pta gene (42.9\%), and numbers 3 and 10 for the yqiL gene $(21.4 \%$ each). The genes of the $S$. aureus isolates were relatively uniform and polymorphic site distribution apparently occurred in conserved regions.
The number of polymorphic sites among the 7 loci in the test samples ranged from $9(\operatorname{arcC})$ to 2 (pta and tpi).

There were 12ST between 9 and 5 control strains and the clinical isolates. The ST5 (ID: 19922) for A13357 SCCmec type IVa and ST105 (ID: 2184) for A30988, SCCmec type II samples were found among our isolates. Furthermore, we observed a new allele of the yqiL gene sequences for A14711 (ID: 1990) and A26151 (ID: 1991) samples. As a result, we describe a new ST belonging to clonal complex 5 (CC5) (ST1176) carrying SCCmec type IVa.

In an attempt to determine the molecular epidemiology of MRSA strains in São Paulo, Brazil, from 2006 to 2007, four isolates (A13357, A14711, A26151, and A30988) were characterized by different molecular typing methods. Two isolates (A13357 and A14711) were characterized as ST1176 in the present study, belonging to CC5 with other Brazilian isolates (ST641, 744, and 1204). The BEC from São Paulo belongs to CC8, together with other Brazilian isolates (ST8, 239, 644, and 250).

MRSA have become a global concern. The dissemination of the MRSA clone probably reflects their ability to cause infection and to persist and spread from one region to another, including different continents (5). The predominance of BEC (SCCmec III) in Brazilian hospitals has been well documented $(3,4,14)$. Recent changes in the profile of clonal MRSA isolated in hospitals have been observed, which have increased the number of MRSA isolates belonging to SCCmec type IV (pediatric clone), as shown in the study by Trindade et al. (15), who reported a $13 \%$ rate of occurrence of MRSA isolates in patients carrying this SCCmec admitted to a São Paulo hospital.

Different typing methods are necessary to determine the molecular epidemiology of MRSA strains and their genetic diversity. Enright et al. (16), by analyzing MRSA samples

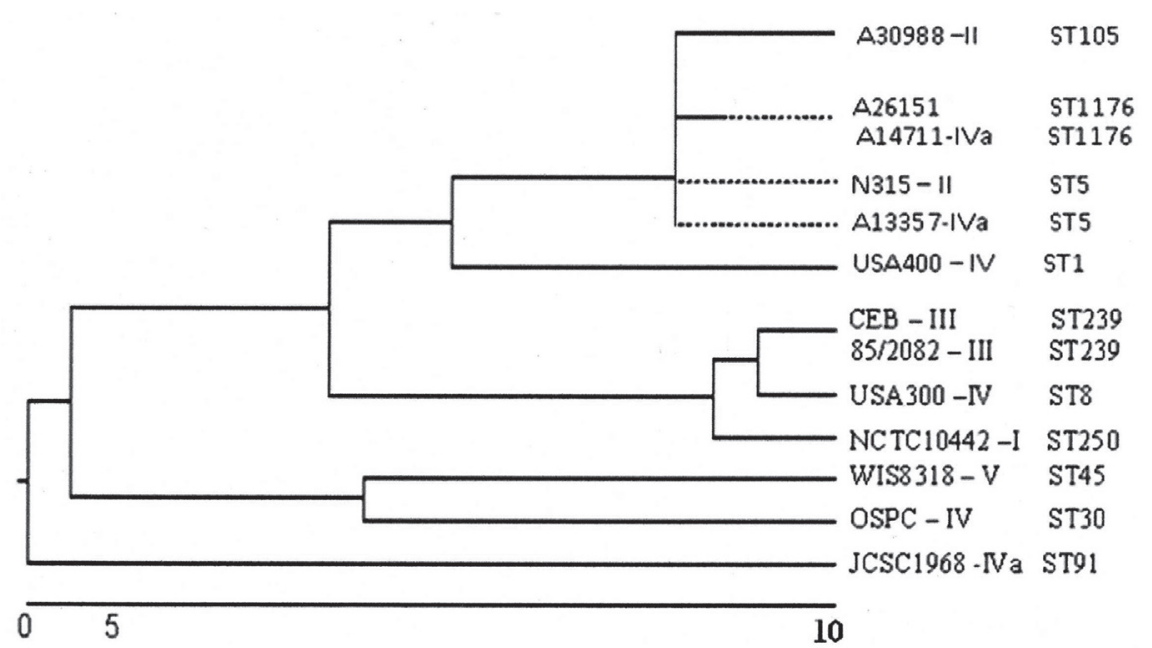

Figure 2. Dendrogram showing the neighbor joining phylogenetic tree, based on the sequence substitution number of 3298 nucleotides. The scale bar below the tree indicates the branch size equivalent to 10 substitutions. 
isolated from hospitals and from the community in different countries using MLST and SCCmec typing, discovered the existence of CCs, allowing the classification of $S$. aureus into evolutionary lineages, resulting in evolutionary models for each lineage (11). These CCs were composed of $S$. aureus samples with the same ST. The comparison of nucleotide sequences of the housekeeping genes between the MRSA samples allowed the establishment of a common ancestor (a methicillin-sensitive Staphylococcus aureus, MSSA). Therefore, samples of MRSA originated from an MSSA by different events of mec acquisition, corroborating the hypothesis of horizontal transmission of the mecA gene (17).

Among the five major MRSA CCs described, the CC5 drift consisted mainly of strains found among the pediatric clones (ST5, SCCmec VI) and NYIJ (ST5, SCCmec II). Strains showing ST8, ST239, ST247, and ST250 are part of CC8, which are found in the Archaic and Iberian clones (SCCmec I) and BEC (SCCmec III) (11). Our results showed that, according to PFGE and MLST, the studied isolates belong to CC5. This CC represents diverse MRSA strains and contains current pandemic clones (11).

Since ST strains have different SCCmec types it is possible that this type was acquired several times, showing

\section{References}

1. Rezende EM, Couto BR, Starling CE, Modena CM. Prevalence of nosocomial infections in general hospitals in Belo Horizonte. Infect Control Hosp Epidemiol 1998; 19: 872876.

2. Richards MJ, Edwards JR, Culver DH, Gaynes RP. Nosocomial infections in medical intensive care units in the United States. National Nosocomial Infections Surveillance System. Crit Care Med 1999; 27: 887-892.

3. Teixeira LA, Resende CA, Ormonde LR, Rosenbaum R, Figueiredo AM, de Lencastre $\mathrm{H}$, et al. Geographic spread of epidemic multiresistant Staphylococcus aureus clone in Brazil. J Clin Microbiol 1995; 33: 2400-2404.

4. Sader HS, Pignatari AC, Hollis RJ, Jones RN. Evaluation of interhospital spread of methicillin-resistant Staphylococcus aureus in São Paulo, Brazil, using pulsed-field gel electrophoresis of chromosomal DNA. Infect Control Hosp Epidemiol 1994; 15: 320-323.

5. Oliveira DC, Tomasz A, de Lencastre $H$. Secrets of success of a human pathogen: molecular evolution of pandemic clones of meticillin-resistant Staphylococcus aureus. Lancet Infect Dis 2002; 2: 180-189.

6. Vogel U, Morelli G, Zurth K, Claus H, Kriener E, Achtman $M$, et al. Necessity of molecular techniques to distinguish between Neisseria meningitidis strains isolated from patients with meningococcal disease and from their healthy contacts. J Clin Microbiol 1998; 36: 2465-2470.

7. Feil EJ, Spratt BG. Recombination and the population structures of bacterial pathogens. Annu Rev Microbiol 2001; 55: 561-590.

8. Clinical and Laboratories Standard Institute (CLSI). Perfor- different genetic compositions in different $S$. aureus strains (11). Thus, we believe that our non-BEC belonging isolates are descendants of a common ancestor, the pediatric clone, first described in Portugal, Poland, Argentina, Colombia, and in New York hospitals' pediatric patients $(18,19)$ or from the pediatric clone NY/Japan that appeared and disseminated in Tokyo and USA hospitals (19). Although in Brazil there was a predominance of BEC in hospitals in several states $(3,4,14,20)$ we found that there was no similarity between this clone and the isolates investigated in the present study. The ST that showed a closely related pattern also had a similar PFGE profile. The present study provides new information about MRSA samples prevalent in São Paulo. The discovery of new strains with allele ST1176 suggests that these bacteria have suffered recent mutations.

Pediatric clone samples seem to be associated with nosocomial infections in other Brazilian cities like Rio de Janeiro, Recife, and in Rio Grande do Sul (14) and São Paulo (15). More studies are needed to characterize MRSA clones not belonging to BEC in Brazil genotypically.

\section{Acknowledgments}

\author{
Research supported by FAPESP and CNPq.
}

mance standards for antimicrobial disk susceptibility tests. 16th Informational supplement. Approved standard M100S17; 9th edn. Wayne: Clinical and Laboratories Standard Institute, 2007.

9. van Soolingen D, Hermans PW, de Haas PE, Soll DR, van Embden JD. Occurrence and stability of insertion sequences in Mycobacterium tuberculosis complex strains: evaluation of an insertion sequence-dependent DNA polymorphism as a tool in the epidemiology of tuberculosis. J Clin Microbiol 1991; 29: 2578-2586.

10. Oliveira DC, de Lencastre H. Multiplex PCR strategy for rapid identification of structural types and variants of the mec element in methicillin-resistant Staphylococcus aureus. Antimicrob Agents Chemother 2002; 46: 2155-2161.

11. Robinson DA, Enright MC. Evolutionary models of the emergence of methicillin-resistant Staphylococcus aureus. Antimicrob Agents Chemother 2003; 47: 3926-3934.

12. Enright MC, Day NP, Davies CE, Peacock SJ, Spratt BG. Multilocus sequence typing for characterization of methicillin-resistant and methicillin-susceptible clones of Staphylococcus aureus. J Clin Microbiol 2000; 38: 1008-1015.

13. Feil EJ, Li BC, Aanensen DM, Hanage WP, Spratt BG. eBURST: inferring patterns of evolutionary descent among clusters of related bacterial genotypes from multilocus sequence typing data. J Bacteriol 2004; 186: 1518-1530.

14. Vivoni AM, Diep BA, de Gouveia Magalhaes AC, Santos KR, Riley LW, Sensabaugh GF, et al. Clonal composition of Staphylococcus aureus isolates at a Brazilian university hospital: identification of international circulating lineages. $J$ Clin Microbiol 2006; 44: 1686-1691. 
15. Trindade de A, Pacheco RL, Costa SF, Rossi F, Barone AA, Mamizuka EM, et al. Prevalence of SCCmec type IV in nosocomial bloodstream isolates of methicillin-resistant Staphylococcus aureus. J Clin Microbiol 2005; 43: 34353437.

16. Enright MC, Robinson DA, Randle G, Feil EJ, Grundmann $\mathrm{H}$, Spratt BG. The evolutionary history of methicillin-resistant Staphylococcus aureus (MRSA). Proc Natl Acad Sci U S A 2002; 99: 7687-7692.

17. Musser JM, Kapur V. Clonal analysis of methicillin-resistant Staphylococcus aureus strains from intercontinental sources: association of the mec gene with divergent phylogenetic lineages implies dissemination by horizontal transfer and recombination. J Clin Microbiol 1992; 30: 2058-2063.

18. Sa-Leao R, Santos Sanches I, Dias D, Peres I, Barros RM, de Lencastre $\mathrm{H}$. Detection of an archaic clone of Staphylo- coccus aureus with low-level resistance to methicillin in a pediatric hospital in Portugal and in international samples: relics of a formerly widely disseminated strain? J Clin Microbiol 1999; 37: 1913-1920.

19. Aires SM, De Lencastre H, Sanches IS, Kikuchi K, Totsuka $\mathrm{K}$, Tomasz A. Similarity of antibiotic resistance patterns and molecular typing properties of methicillin-resistant Staphylococcus aureus isolates widely spread in hospitals in New York City and in a hospital in Tokyo. Japan Microb Drug Resist 2000; 6: 253-258.

20. de Miranda OP, Silva-Carvalho MC, Ribeiro A, Portela F, Cordeiro RP, Caetano N, et al. Emergence in Brazil of methicillin-resistant Staphylococcus aureus isolates carrying SCCmecIV that are related genetically to the USA800 clone. Clin Microbiol Infect 2007; 13: 1165-1172. 